\title{
Polyethylene glycol submucosal irrigation: a novel approach to improve visibility during endoscopic submucosal dissection
}

Authors

Institutions
Vitor Arantes ${ }^{1}$, Takashi Toyonaga ${ }^{2}$, Elias Alfonso Forero Piñeros ${ }^{3}$

${ }^{1}$ Faculdade de Medicina da Universidade Federal de Minas Gerais - Alfa Institute of Gastroenterology, Belo Horizonte, Brazill

${ }^{2}$ Kobe University Hospital - Endoscopy, Kobe, Japan

Hospital de la Policia - Endoscopy, Bogota, Colombia submitted 21. April 2014 accepted 3. June 2014

\section{Bibliography Dol http://dx.doi.org/ 10.1055/s-0034-1377444 Published online: 16.7.2014 Endoscopy International Open 2014; 02: E193-E195 (c) Georg Thieme Verlag KG Stuttgart $\cdot$ New York E-ISSN 2196-9736 \\ Corresponding author T. Toyonaga, M.D. Department of Endoscopy Kobe University Hospital 7-5-1 Kusunoki-cho Chuo-ku \\ Kobe \\ 5500-0017 - Japan Fax: +81-78-3826309 toyonaga@med.kobe-u.ac.jp}

In order to expand the availability of endoscopic submucosal dissection (ESD), measures to facilitate the procedure are necessary. When bleeding occurs, the examiner's field of vision is critically impaired, and ESD becomes less efficient and more hazardous because of the presence of submucosal hematoma and covered blood clot. We

\section{Introduction}

$\nabla$

Endoscopic submucosal dissection (ESD) has been developed to enable the endoluminal en bloc resection of superficial gastrointestinal neoplasms, thus producing an adequate specimen for a reliable histologic assessment. Several devices and tools have been proposed to make it possible to perform ESD safely and in an effective manner [1-3]. Despite these advances, ESD still is a challenging and time-consuming procedure. In order to promote the widespread availability of ESD, measures to facilitate and standardize the procedure are required. One of the main problems associated with ESD is hemorrhage control $[4,5]$, and a correct vascular approach is key to preventing bleeding and improving the submucosal dissection [6,7]. Whenever bleeding occurs, the examiner's field of vision is critically impaired, and ESD becomes less efficient and more hazardous. We propose a simple and widely available measure to improve the visibility of the submucosal space when bleeding occurs during ESD: polyethylene glycol (PEG) irrigation.

\section{Case Report \\ $\nabla$}

A 70-year-old man was referred to Kobe University Hospital, Kobe, Japan, for endoscopic treatment of a laterally spreading tumor (LST) in the left side of the colon. He denied co-morbidities and the consumption of anticoagulants or antithrombotic agents. After discussion with the patient, he propose the use of polyethylene glycol (PEG) irrigation as a simple and effective measure to improve visibility during submucosal dissection, particularly when bleeding occurs. PEG irrigation facilitates further dissection by allowing a better recognition of the submucosal fibers and muscularis propria layer.

agreed to undergo ESD and signed a consent form covering all the components of ESD: preparation, the surgical procedure with related endoscopic maneuvers, and postoperative care.

The novel use of PEG irrigation for submucosal cleansing is illustrated in the figures. Fig. 1 shows a large, flat-elevated, homogeneous, granular-type LST. After mucosal incision and submucosal dissection with a $1.5-\mathrm{mm}$ ball-tipped FlushKnife (Fujifilm, Tokyo, Japan), we observed massive bleeding from large vessels, which was stopped with a coagulation grasper. $\bullet$ Fig. 2 shows the condition of the field after control of the bleeding and irrigation with water. Submucosal hematoma and covered blood clot still interfere with clear vision and disturb the orientation of the submucosal layer. Irrigation with saline solution was ineffective. Submucosal hematoma and covered clot were cleared by flushing with PEG (Niflec; Ajinomoto Pharmaceuticals, Tokyo, Japan) in a $30-\mathrm{mL}$ syringe up to three times through the working channel, and the submucosal transparency was restored ( $\nabla$ Fig.3). A clearer view of the surface of the muscular layer through the transparent submucosa enabled further submucosal dissection safely. PEG irrigation also proved to be useful by making it possible to identify the oozing vessel precisely, facilitating direct hemostasis. A complete en bloc resection ( $\bullet$ Fig.4) was obtained successfully in 80 minutes, and the specimen was fixed for histologic assessment ( $\bullet$ Fig. 5). The patient experienced an uneventful recovery and was discharged home after 4 days of in-hospital observation. No adverse event related to the use of 


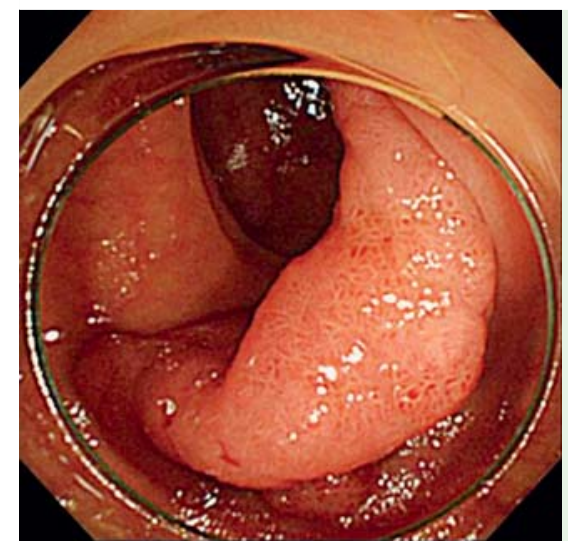

Fig. 1 Large,

flat-elevated, homogeneous, granular-type laterally spreading tumor in the ascending colon of a patient referred for endoscopic submucosal dissection.

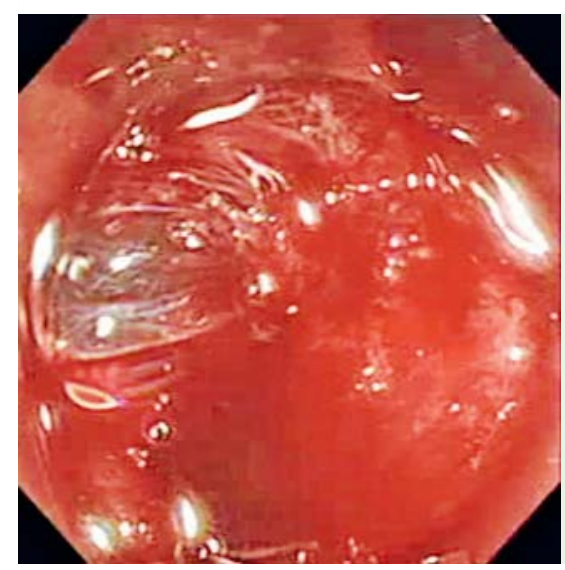

Fig.2 After control of bleeding and flushing with water, submucosal hematoma and covered blood clot still impair visibility.

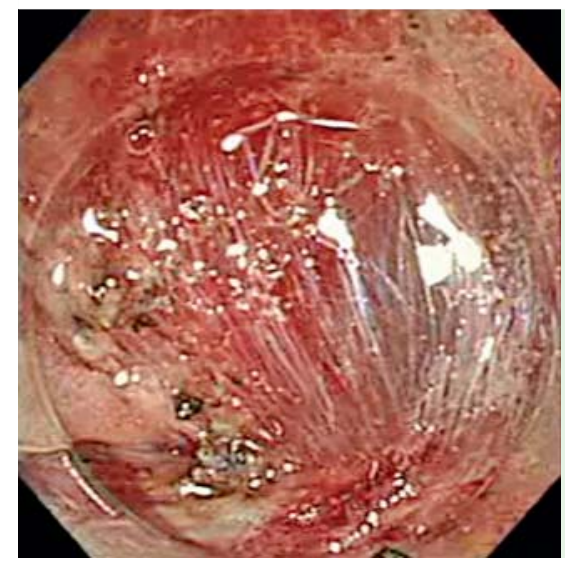

Fig. 3 Submucosal blood clot and hematoma have been cleared by polyethylene glycol irrigation. Note the restored transparency, allowing a better view of the submucosal layer and surface of the muscular layer.

PEG as an irrigation method was noted. Histology showed a tubulovillous adenoma with high grade dysplasia, no lymph node or vascular invasion, and free horizontal and vertical margins.

\section{Discussion}

\section{$\nabla$}

PEG is a polymer of ethylene oxide with a wide range of applications in medicine [8]. It has been used in gastroenterology mainly as a laxative for bowel preparation before colonoscopy or the management of chronic constipation. PEG also is safely used as a pharmaceutical ingredient in ointments, suppository formulations, creams, lotions, lubricants, coating materials of tablets, and solubilizers for injection [8]. This polymer has a low level of toxicity, and when absorbed, it is eliminated through hepatic or

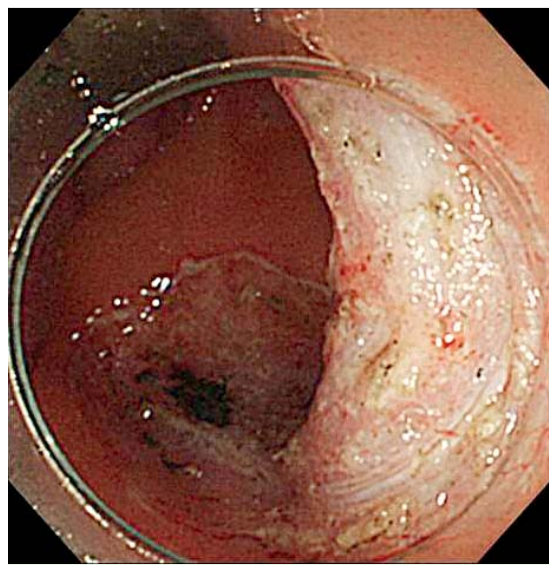

Fig. 4 Final result: a complete en bloc resection.

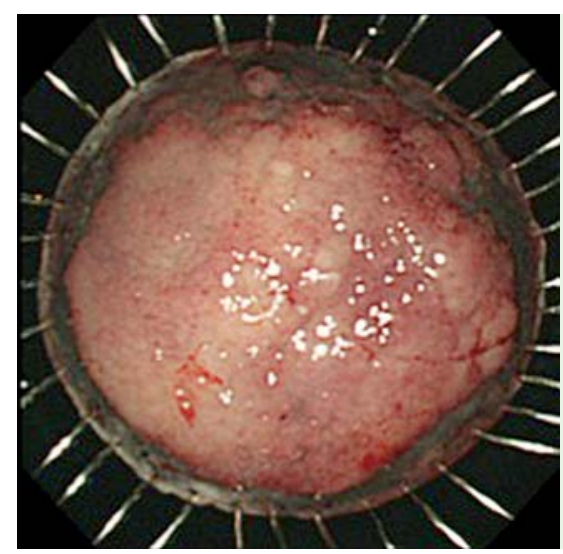

Fig. 5 The specimen has been fixed for histologic assessment.

renal pathways [8]. Previously, the only application of PEG related to endoscopic resection has been reported in experimental settings, as a submucosal injectant [9].

PEG irrigation is a simple and effective means to improve visibility in submucosal dissection, particularly when bleeding occurs; it facilitates ESD by allowing a better recognition of the submucosal fibers. As demonstrated in this case report, PEG irrigation improved the field of view substantially and made it possible to continue ESD in a safer fashion. When bleeding takes place during ESD, hemostatic control with coagulation forceps or the knife blade is immediately attempted, together with water irrigation as needed. However, when the control of bleeding takes a long time and the field is occupied by blood or clots, irrigation with water is not very efficient, and visibility is compromised. In such situations, proceeding with blind hemostatic maneuvers without a clear vision of the submucosal space is hazardous and may lead not only to a failure of bleeding control but also, most importantly, to accidental damage and perforation of the muscularis propria layer. It is in this situation that we recommend cleaning the submucosal space by means of irrigation with PEG.

We assume that the effectiveness of PEG irrigation to clear blood clots is due to its property as a dispersing agent; however, the mechanism of this effect remains to be clarified. In addition, we have noted that PEG irrigation is also useful when persistent bowel residual covers a colonic flat lesion. PEG irrigation makes it possible to clean the lesion surface very effectively, optimizing conditions for chromoendoscopy and magnifying the examination.

In conclusion, we propose a novel use for PEG during endoscopic procedures, aimed at cleaning the submucosal space of blood clots and improving visibility for submucosal dissection whenever bleeding occurs and cannot be controlled promptly. Further 
studies are required to reproduce our experience in a large number of patients and to confirm the reliability of this approach.

\section{Competing interests: None}

\section{References}

1 Toyonaga T, Nishino E, Hirooka $T$ et al. Use of short needle knife for esophageal endoscopic submucosal dissection. Dig Endosc 2005; 17 : $246-252$

2 Toyonaga T, Man-I M, Fujita T et al. The performance of a novel ball-tipped Flush knife for endoscopic submucosal dissection: a case-control study. Aliment Pharmacol Ther 2010; 32: 908-915

3 Uraoka T, Saito Y, Yahagi N. What are the latest developments in colorectal endoscopic submucosal dissection? World J Gastrointest Endosc 2012; 4: $296-300$
4 Park CH, Lee SK. Preventing and controlling bleeding in gastric endoscopic submucosal dissection. Clin Endosc 2013; 46: 456- 462

5 Yoshida N, Yagi N, Nayto Y et al. Safe procedure in endoscopic submucosal dissection for colorectal tumors focused on preventing complications. World J Gastroenterol 2010; 16: 1688 - 1695

6 Toyonaga T, Nishino E, Hirooka T et al. Intraoperative bleeding in endoscopic submucosal dissection in the stomach and strategy for prevention and treatment. Dig Endosc 2006; 18: $123-127$

7 Toyonaga T, Nishino E, Dozaiku T et al. Management to prevent bleeding during endoscopic submucosal dissection using the flush knife for gastric tumors. Dig Endosc 2007; 19: $14-18$

8 Ishihara $\mathrm{H}$. Current status and prospects of polyethyleneglycol-modified medicines. Biol Pharm Bull 2013; 36: 883-888

9 Polymeros D, Kotsalidis G, Triantafyllou K et al. Comparative performance of novel solutions for submucosal injection in porcine stomachs: an ex vivo study. Dig Liver Dis 2010; 42: 226-229 